\title{
Nuclear Power Plant Instrumentation Fault Detection Using Fuzzy Logic
}

\author{
Keith E. Holbert and Kang Lin \\ School of Electrical, Computer and Energy Engineering, Arizona State University, Tempe, AZ 85287-5706, USA \\ Correspondence should be addressed to Keith E. Holbert, holbert@asu.edu
}

Received 30 May 2012; Revised 25 July 2012; Accepted 9 August 2012

Academic Editor: Yan Yang

Copyright ( $) 2012$ K. E. Holbert and K. Lin. This is an open access article distributed under the Creative Commons Attribution License, which permits unrestricted use, distribution, and reproduction in any medium, provided the original work is properly cited.

Economic constraints are driving the electric power industry to seek improved methods for monitoring, control, and diagnostics. To increase plant availability, various techniques have been implemented in industry to assess equipment condition to prevent system inoperability. The availability of a large number of measured signals and additional component information and the increasing number of signal processing options to analyze sampled data motivate the assimilation of such diverse information into a plantwide condition monitor. The use of fuzzy logic is described herein for the purpose of performing the decision making regarding the system status and the possible need for component maintenance. Fuzzy-logic-based diagnostic monitoring is applied to data acquired from instrumentation within operating facilities.

\section{Introduction}

Competition due to the deregulation of the electric power industry has provided additional motivation for power plant owners to implement predictive maintenance (PM) programs. Equipment health monitoring is known by a variety of related endeavors including condition-based and reliability-centered maintenance. To accomplish the PM without interrupting equipment operation necessitates the use of online monitoring tools for signature analysis. Those signatures, in turn, must be scrutinized to ascertain whether the system or component is trending toward a failure condition. The originating signals are often the result of stochastic (random) processes.

Figure 1 illustrates three basic approaches to equipment maintenance. Corrective (or reactive) maintenance is taken only after the component has failed. To avert breakdown, preventive approaches involve anticipatory actions based on a schedule or prediction. Scheduled maintenance, which may involve inspections and/or preemptive replacements, can be performed on either calendar or equipment use bases. Predictive maintenance is initiated because of a detected onset of equipment malfunction or failure. Reliability-centered maintenance incorporates all three approaches while considering the importance of the equipment to the facility mission and is generally based upon failure modes and effects analysis.

The capability to detect faults and to replace the components just prior to failure is desired by industry. By doing so, the consequences of unexpected equipment failures can be avoided. Online component monitoring can yield higher availability, extended life, and reduced costs. Incipient failure detection serves not only to avoid catastrophic failure, but also to assist in planning corrective actions (i.e., preventive maintenance). Incipient failure detection has the ability to assist in achieving condition-based maintenance objectives.

Many fault detection and isolation (FDI) techniques have been developed over the years. The approach presented in this paper seeks to build upon proven FDI methods for sensor validation. Whereas the vast majority of research focuses on a specific diagnostic approach, this work seeks a means by which the diverse diagnostic information provided by multiple techniques may be integrated-fuzzy logic is chosen as the agent to achieve this data fusion.

This paper begins with a brief survey of the applications of fuzzy logic to diagnostics and power plants. The next section then describes the philosophy behind the fuzzy logic diagnostic monitoring (FLDM). This is followed by a description of how the FLDM can be implemented for 


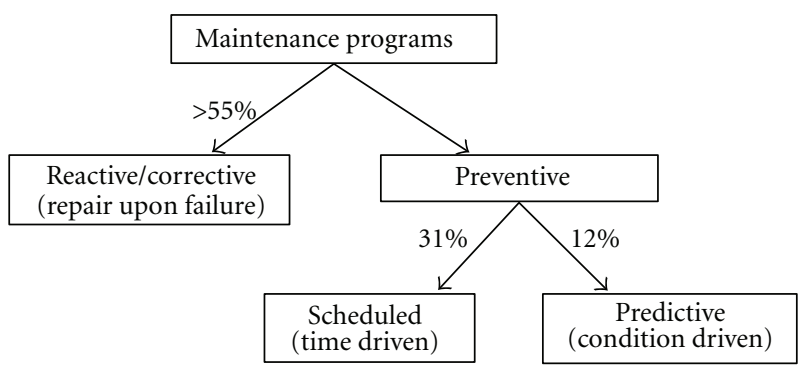

FIGURE 1: Equipment maintenance approaches. The percentages represent the categorization of maintenance program in the USA and are taken from [8] based on a survey in 2000.

condition monitoring. Analysis results from applying the fuzzy logic health monitoring to data acquired from nuclear reactor systems are presented in conjunction with the methodology description.

\section{Applications of Fuzzy Logic}

A popular application of fuzzy logic (FL) has been in the area of control. Implementations of fuzzy control in electrical power stations include solar thermal power plants [1, 2], nuclear unit steam generator level control [3-5], and groundwater pumping at a coal-fired unit [6]. As an example bridging control and diagnostics, Holmes and Ray studied control system development for a fossil power plant in which a fuzzy-logic-based controller was tasked with making judgments between system dynamic performance versus structural durability in critical components [7].

As this paper is focused on power plant diagnostics, the remainder of this section briefly surveys some of the fuzzy logic applications in these domains. Choi et al. developed an online fuzzy expert system to provide the operator with uncluttered alarm displays and systemwide failure information during anomalous conditions and carry out alarm prognosis to warn the operator of process abnormalities in nuclear power plants [9]. Holbert et al. applied fuzzy logic to intrusion detection in supervisory control and data acquisition (SCADA) systems [10]. Pareek and Kar presented a successful demonstration of the application of a type 2 fuzzy logic system to predict the compressor discharge pressure of a power plant gas turbine [11]. Köppen-Seliger et al. used a fuzzy logic rule base to evaluate the residuals obtained by any available analytical knowledge from several subsystems to perform fault diagnosis for the high-pressure preheater of a power plant [12]. In the work of $\mathrm{Na}$ et al., a smart software sensor using a fuzzy model was developed to perform accurate online estimation of the feedwater flow rate and also to monitor the status of the existing hardware sensors for pressurized water reactors [13]. Fuzzy-logicbased diagnostics have been applied to a variety of electric generating stations including a hybrid fuel cell—gas turbine facility [14] and a combined cycle power plant [15].

Fuzzy logic has also been applied to component and process diagnostics in problems of a general nature [16]. For instance, Mendonça et al. employed a fuzzy model to perform FDI for an industrial servoactuated pneumatic valve [17]. Yui and Lee utilized fuzzy set theory to integrate quantitative information into a qualitative model-based diagnostics system [18]. Aubrun et al. tested an FL-based evaluation and reconfiguration method for actuator failures using simulation data from a thermal plant [19]. In their work, FL was used for both residual evaluation and to reconfigure the control system using fuzzy rules. Regarding the fault isolation, they concluded that FL provides better results in terms of the robustness of the models as compared with the classical methods [19]. Amin et al. applied fuzzy inference systems, knowledge fusion, and feature extraction to create a robust health monitoring system for the determination and classification of pump degradation [20]. For creating a failure detection system destined for complex processes such as a chemical plant, Vaija et al. proposed the use of a multilevel fuzzy system [21]. Mechefske applied fuzzy logic techniques to classify frequency spectra that represent various rolling element bearing faults [22]. Uriu et al. successfully expressed the quench margin of a superconducting magnet system in terms of a "danger factor" that is generated by incorporating data gathered from numerous sensors [23].

A few researchers have also promoted FL in the context of instrumentation fault detection. Application of FL to signal validation appears to have been first proposed by Heger et al. in 1993 [24]. As they stated, fuzzy logic is useful for instrument fault detection, as it possesses the advantage of transforming linguistic information to numerical values for processing and then later back to the linguistic domain $[25,26]$. Mourot et al. suggested the use of fuzzy pattern recognition for gross error detection problems [27] as may be encountered when utilizing parity equations to generate residuals. Sauter et al. investigated the use of FL to diagnose sensor and actuator faults in a simulated mechanical system. In particular, they evaluated residuals in an adaptive manner, and they used an FL technique to diagnose fault signatures from a dedicated observer scheme [28]. In addition, Hines et al. used FL to model and estimate process states as part of an adaptive neural-fuzzy inference system intended to perform instrument channel calibration verification [29]. Other hybrid implementations of FL for sensor validation include the coupling of FL with state estimation techniques [30].

As sensors provide the means by which operators and control systems regulate systems, the field of instrumentation fault detection and isolation has seen many studies over the years. For those readers seeking to review the scientific literature in the field of FDI, see the recent review papers [31, 32], reports [33], and books [34, 35].

\section{Fuzzy Logic Diagnostic Monitoring}

Predictive maintenance programs generally employ signal processing techniques to perform condition monitoring on particular plant equipment and systems. Such efforts have the potential to draw upon a plethora of digital signal processing methods which have been developed and can be targeted toward specific fault signatures. These approaches are challenged to perform FDI and anomaly characterization, 


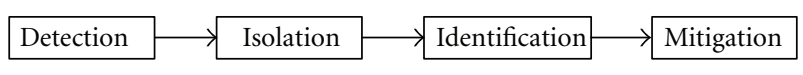

Scheme 1

where these two tasks are listed in order of increasing difficulty. Furthermore, some FDI algorithms provide straightforward diagnostics with applicability to a variety of anomalies while other FDI approaches are complex computational tools dedicated to the analysis of specific failure modes. As a case in point, some methods are more useful for evaluating abrupt faults while others are intended for incipient failures. This situation leads to condition monitors that are often dedicated to a single piece of equipment and incorporating software with limited applicability in terms of the potential failure modes that a component may experience. Small electric utilities and individual generating units cannot be expected to employ a dedicated staff that is cognizant of and conversant with all the available FDI methods. Consequently, an operational aid in the form of an intelligent monitoring system is needed. This state of affairs is an impetus for the development of the rule-based fuzzy logic diagnostic monitoring (FLDM) to be described here.

3.1. Philosophical Approach. One facet of the philosophical approach taken in developing the FLDM was to consider the methodology often taken when a person is ill. Typically, a physician first employs a variety of simple measures (e.g., body temperature) and afterwards may order additional diagnostic tests (e.g., X-ray) to be performed based on the initial evaluation of the patient. This thought process led to an additional FLDM attribute in a further effort to provide an effective health monitor, specifically the incorporation of a knowledge (data) base of information concerning the equipment operational history including manufacturer reliability data and other maintenance records. Similar to patient records, baseline signatures (when the equipment is functioning in a normal, fault-free manner) are acquired and stored for future reference and comparisons.

When performing FDI, the four stages, listed in order of increasing difficulty, are as follows (see Scheme 1)

(1) detection that an actual fault has occurred,

(2) isolation of the fault components/devices,

(3) identification of the fault type and/or cause, and

(4) mitigation of the fault (e.g., repair).

The plantwide fuzzy logic health monitoring employs multiple signal processing modules to perform fault detection, isolation, and characterization. Operational data signals are sampled from existing and retrofitted sensors throughout the power plant. The modular architecture of the FLDM then allows signal analyses in parallel, that is, the health of a given component or system can be assessed using multiple independent signal processing techniques. As depicted in Figure 2, the FLDM uses a two-stage approach in which the first phase employs the more common techniques for robust fault detection. Subsequently, the second stage algorithms,

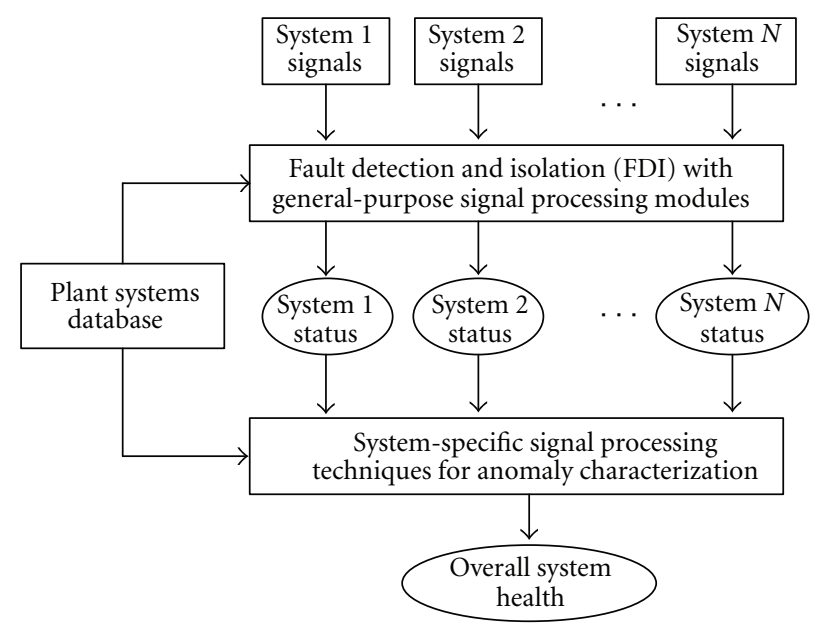

FIgURE 2: Overall scheme of the fuzzy logic diagnostic monitoring.

which are intricate in nature, are intended to characterize the anomaly. The characterization effort is critical to determining the proper maintenance response.

Creating the FLDM then requires the selection of suitable signal processing modules. It must be recognized that each approach has its advantages and disadvantages. Carnero noted, in the context of a screw compressor predictive maintenance program, that the most suitable diagnostic techniques must be selected or the PM program may fail [36]. Furthermore, process conditions (e.g., transient changes and the particular steady-state operating point) can adversely affect the ability of some signal processing techniques to accurately analyze the data. Of course, reliable commercial implementations favor those methods with lowmissed and false alarm rates. Instantaneous failure detection using single-observation conclusions is appropriate only in those cases in which gross failure is experienced. Alternatively, long-term monitoring is required to identify slowly developing faults such as those anticipated in a predictive maintenance application. In order to improve the overall reliability of the health monitoring, we promote the use of multiple methods to address the diverse failure mechanisms (categories) and the implementation of multiple techniques within a failure category to improve the fault detection.

A salient feature of the approach presented in this paper is that it builds upon proven fault detection and isolation techniques. A challenge to an integrated condition monitoring system is the assimilation of the diverse results from the signal processing modules into a final status decision. Over time, experience may demonstrate the need to remove a module due to poor performance, or if a new state-of-theart technique becomes available, then it can be inserted into the existing integrated health monitor. To accomplish this, we employ a rule-based fuzzy logic decision maker.

Many methods are geared toward the detection of a single failure mode and, therefore, lack the robustness required for any practical application. Furthermore, various equipment and component failure modes exist. Two additional failure cases which must be considered are the possibilities of 


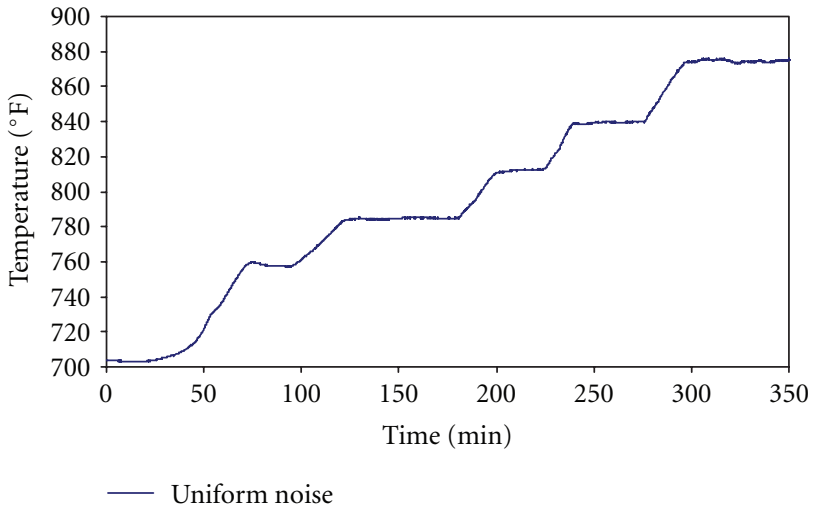

(a) Little change in noise

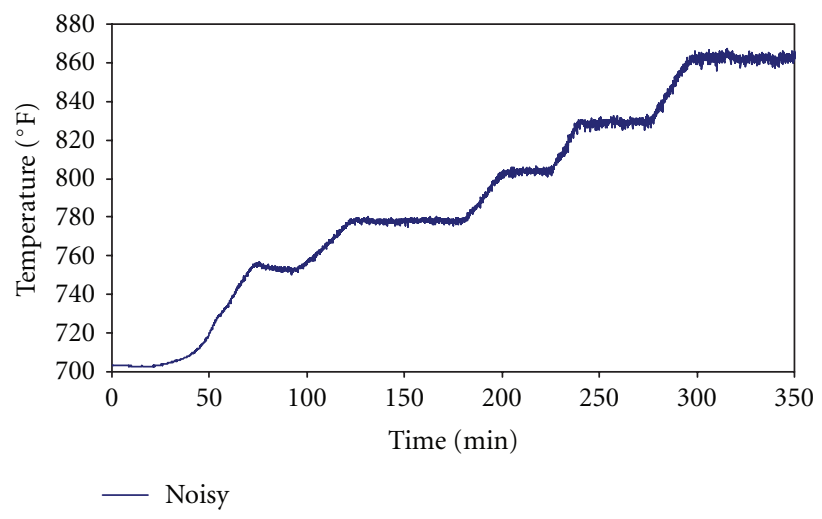

(c) Significant increase in noise

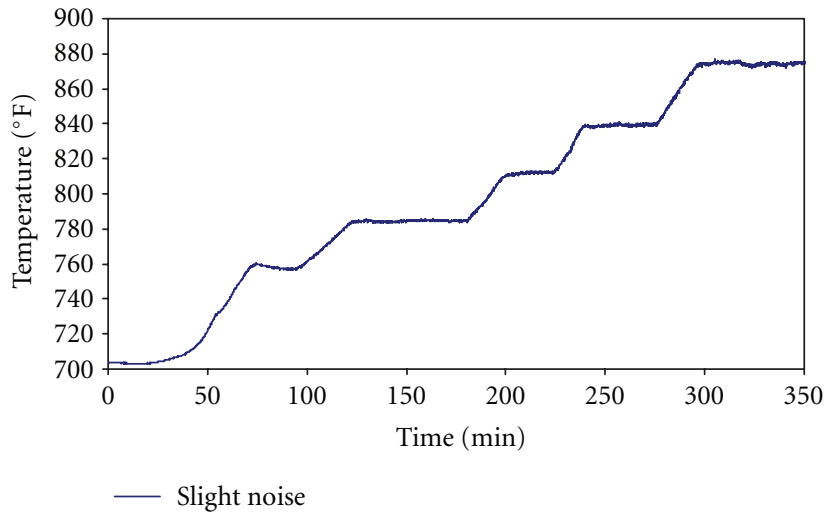

(b) Slight increase in noise

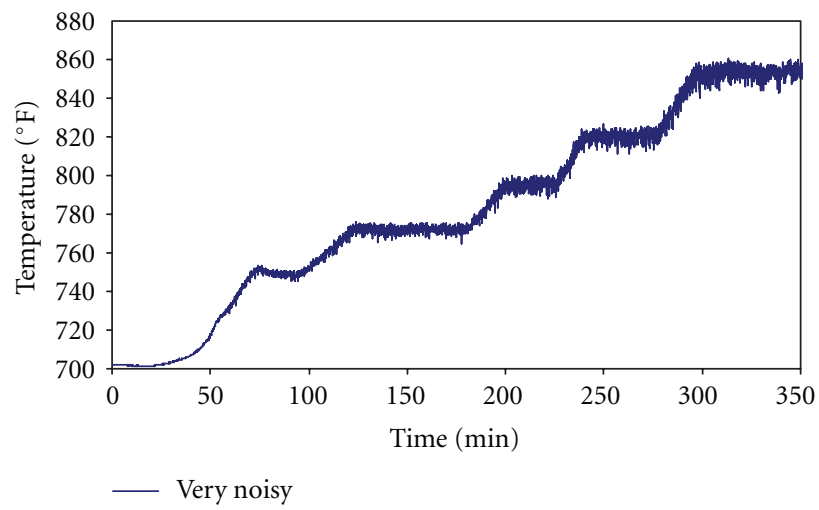

(d) Considerable increase in noise

FIGURE 3: Four temperature sensors with varying amounts of signal noise as the reactor power is raised from $0 \%$ to $100 \%$.

multiple failures (via the same or different failure modes) and common-mode failure. Detection of multiple failure modes has obvious advantages; however, the more complex the failure mode, the more difficult it is to detect and the further the fault detection scheme tends into the area of process fault diagnostics.

3.2. Stage 1 Signal Processing Modules. To achieve wide applicability throughout an entire industrial plant, such as a nuclear generating station, general-purpose signal processing techniques are needed. These signal processing modules provide the initial analyses of plant data to ascertain whether an anomaly exists. The more in-depth analyses can be performed, if desired, to more definitively characterize the problem.

To illustrate the use of the general-purpose signature analysis methods, consider the four temperature signals shown in Figure 3. These signals were obtained from the upper plenum of the Experimental Breeder Reactor II during a startup from $0 \%$ to $100 \%$ full power. The first signal (Figure 3(a)) visually shows a uniform amount of noise regardless of the power level; however, the other three signals exhibit increasing levels of noise as the reactor power rises, with the last temperature reading (Figure 3(d)) being especially noisy. The signal noise could be due to sensor maloperation or a process irregularity. Or perhaps the
TABLE 1: A simple set of rules for signal noise assessment.

\begin{tabular}{lccc}
\hline Rule & RMS value & Zero-crossing rate & Signal noise status \\
\hline 1 & Low & $\overline{\text { Infrequent }}$ & Normal \\
2 & Ordinary & Infrequent & Noisy \\
3 & Zero & Infrequent & Suspect \\
4 & Low & Infrequent & Suspect \\
5 & Elevated & Frequent & Very Noisy \\
\hline
\end{tabular}

increased noise is perfectly normal and it is the signal without increased noise that is actually abnormal. The FL decision maker must combine the signal analysis results with expert derived rules to ascertain the correct diagnosis.

Consider a fuzzy noise detector that utilizes only two inputs: the rms and zero-crossing rates of the signals. The rms and zero-crossing rate are first measured and individually fuzzified. The rms value may be fuzzified into zero, low, ordinary and elevated values. The zero value is necessary to account for situations such as signal saturation and loss of signal. The zero crossing rate (ZCR) is similarly fuzzified into infrequent, regular, and frequent. For our first example, a simple rule set is produced and delineated in Table 1. Rules 3 and 4 account for situations such as signal saturation and loss of signal. 


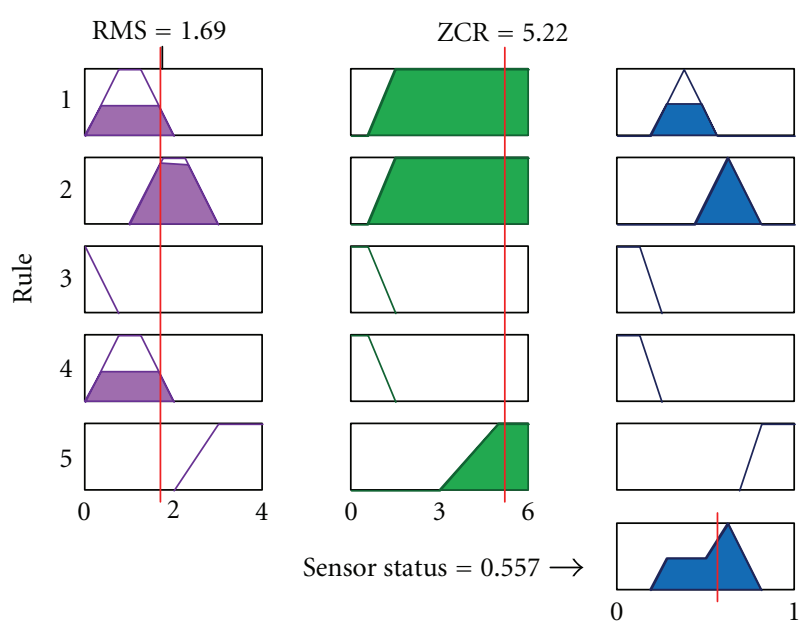

Figure 4: Application of fuzzy noise detector to the third temperature signal.

In the case of the second temperature sensor (Figure $3(\mathrm{~b}))$, the rms value is computed as $0.80^{\circ} \mathrm{F}$ and the ZCR is 2.75 per min at around $t=350$ mins. The rms value is fuzzified and found to have a membership of 1.0 in the low group. Likewise, the ZCR is fuzzified and determined to have a unity membership in the infrequent (note: the overline is read as not infrequent) class. After applying the rules and performing the centroid defuzzification, the overall signal noise status is normal as only Rule 1 is activated, even though the human perception based on Figure 3(b) might be to consider it noisy.

The results for the third and fourth signals are similar, except that the fourth signal is classified as very noisy and the third signal is deemed simply noisy. The $\sigma=1.69^{\circ} \mathrm{F}$ of the third temperature produces memberships of 0.39 and 1.0 in the low and ordinary groups, respectively; the ZCR = $5.22 / \mathrm{min}$ activates both the frequent and infrequent to a degree of unity as seen in Figure 4. The diagnostic result for the third signal is noisy even though both Rules 1 and 2 are triggered.

The analyses of this example have been performed on a single signal, and the rms and ZCR are fuzzified according to the baseline signal features that are stored in the plant database, which is described later. Although this example has shown analyses based on examining a single sensor without regard to the behavior of other similar signals, the features from redundant and related signals can also be compared to further enhance the confidence of the result. Moreover, such comparisons aid in resolving whether the noise is the consequence of an equipment anomaly or a process abnormality.

As an example of the interdependency between process and equipment diagnostics, consider the spike in the reactor core exit temperature signal shown in Figure 5. Such an occurrence requires an evaluation as to whether this impulse is an actual process change or an erroneous reading. Rateof-change limit checking can be used in this situation to ascertain that, in fact, such a physical change is improbable,

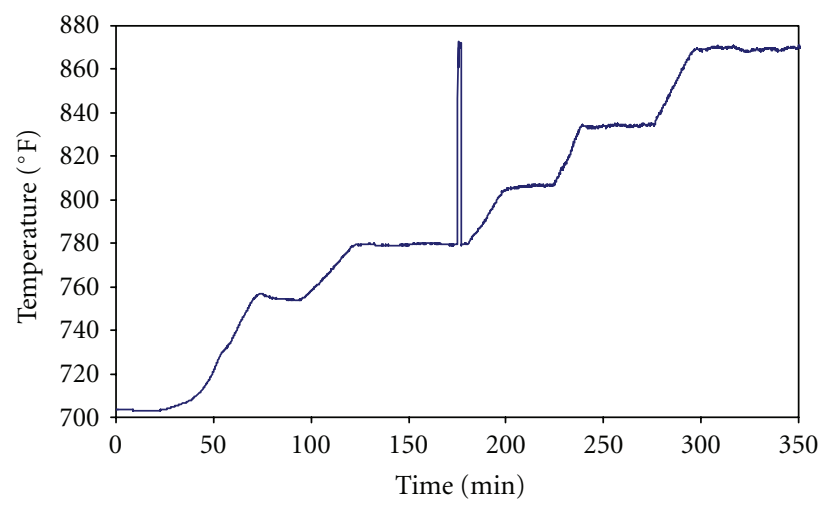

FIGURE 5: Reactor core exit temperature measurement with spurious behavior during startup operations.

but not impossible. Nevertheless, this possibility forms the basis of several rules which depend on the variable of interest and the magnitude of the rate of change as defined by fuzzy membership functions. Furthermore, additional information can be incorporated in the decision-making process by looking at other related process variables. For instance, such a large temperature rise would require a significant increase in power and/or a loss of heat rejection capability as might be experienced during a loss of coolant flow. This situation demonstrates the need for the fuzzy rule knowledge base to discriminate equipment malfunction from process abnormalities.

Rudimentary signatures such as these have wide applicability across plant systems. The failure modes addressable by fundamental signal descriptors include spikes, drift, bias, noise, and stuck. Pattern recognition and additional digital signal processing modules employing advanced time and frequency domain techniques could also be included within the diagnostician, but those would generally be the forte of the characterization stage. Besides random signal analysis, deterministic test results can also be included as input to the condition monitor, for example, machine lubricating oil and wear particle analyses, but again those represent indepth and system-specific methods. The effort in realizing an effective diagnostic monitor then becomes the analysis of the acquired data in order to determine whether the particular system or component is exhibiting characteristics that warn of incipient failure.

3.3. Diagnostic Monitoring. Process data fusion, analysis, and interpretation represent key barriers to achieving better equipment health monitoring. Results have shown that fault detection must be accomplished by using diverse signal processing techniques to perform robust signal analysis; however, these methodologies should be melded into a cohesive fault detection scheme rather than combined in a parallel scheme that permits no crosstalk between the signal processing techniques. A rule-based fuzzy logic approach allows the combination of information from multiple domains. 


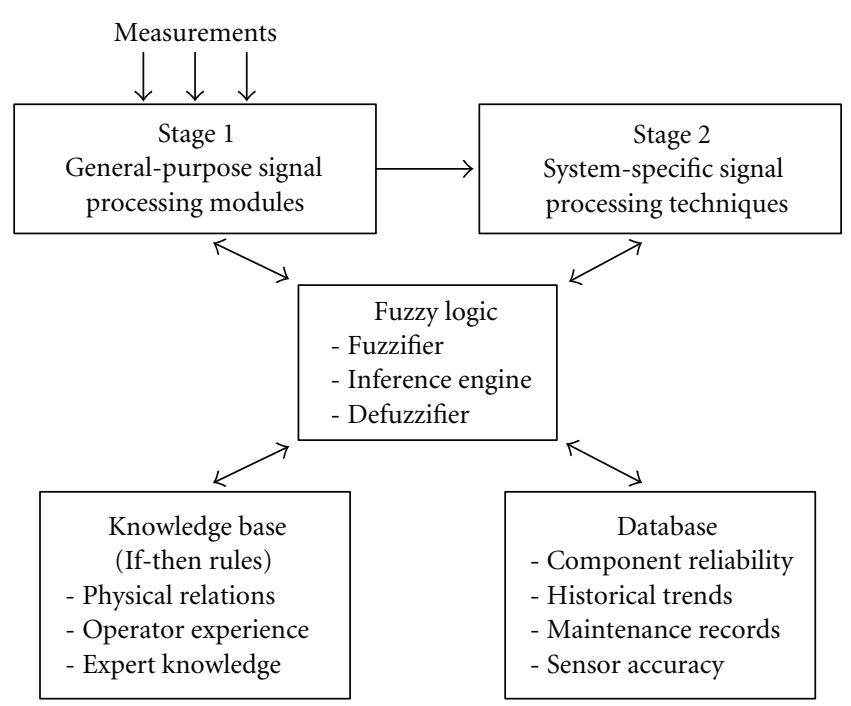

Figure 6: Connection between the fuzzy logic decision making and the signal processing modules and other related information of importance.

General signal processing techniques are useful but indepth equipment condition monitoring requires equipmentspecific analysis algorithms. As an example, consider the authors' health diagnostics of pressure sensing lines in nuclear power plants $[37,38]$. For such instrument line diagnostics, the power spectral density of the pressure fluctuations (noise) is determined and compared to transfer function based models of normal and anomalous conditions such as line blockage, gas entrapment, and leakage [39]. In the end, however, all of these results must be combined via a higher-level decision-making module - this is the role of the FLDM.

Fuzzy logic is employed in a decision-making capacity (see Figure 6) for system diagnostics using signal processing modules. By keeping the diagnostic monitor flexible with respect to the applicable types of equipment and systems, the FLDM can be applied on a plantwide basis. The system can also be expanded through the addition of other FDI methods (such as Kalman filtering) to process the collected data and information.

In encoding the FLDM, we recognize that plant personnels have an advantage over FDI methods in terms of their personal experience and the human capability to assimilate a wide range of information and new data. In contrast, computers have an edge in being able to process such information faster than their human counterparts can. Fuzzy logic, as an artificial intelligence tool, can take advantage of the plant operators' experience and the rapid data processing capability of computers. The numerical results of the various signal-processing modules are fuzzified for processing by the FLDM, which incorporates the intellect of the engineeranalyst into the fuzzy rules. The rule-based fuzzy system created here uses the Mamdani fuzzy inference process. The fuzzy rules and weighting factors are tailored to the particular equipment and signal processing techniques. For example,
TABLE 2: Plant database.

\begin{tabular}{|c|c|}
\hline Information category & $\begin{array}{l}\text { Specific information stored in the } \\
\text { database }\end{array}$ \\
\hline Instrumentation & $\begin{array}{l}\text { List of measured variables, sensors, } \\
\text { accuracy }\end{array}$ \\
\hline Baseline signatures & $\begin{array}{l}\text { NRMS, skewness, kurtosis, } \\
\text { zero-crossing rate }\end{array}$ \\
\hline Equipment specifications & Manufacturer, model number \\
\hline Maintenance records & $\begin{array}{l}\text { Dates of and technician performing } \\
\text { the last calibration and most recent } \\
\text { repair; reliability history }\end{array}$ \\
\hline
\end{tabular}

a zero weighting factor can be used to prohibit a particular module from analyzing data from a given component while still making the module available for examining other plant systems. The weights are chosen to reflect the confidence by which a given signal processing module can properly identify a given failure mode within that particular equipment/ system. The computational overhead for the fuzzy system is such that signal analysis can be accomplished in real time. The modular architecture allows the insertion of new stateof-the-art techniques when they are deemed useful to electric generating stations.

A rule base is created allowing the human perception of the situation to be represented mathematically. The rule base of the fuzzy logic approach is generic in the sense that the rules are oblivious to the particular state variable being measured. Fuzzy set operations are applied in conjunction with the rules. Finally, a defuzzification scheme is used to determine the centroid location and, therefore, the signal status. Although the signal processing modules may be applied to all the collected data, this is not to say that the weighting factors are the same for all plant components and systems. Whereas the various techniques generally utilize strict numeric boundaries during the fault detection, the monitor described here relaxes the hard boundaries by utilizing fuzzy set theory. As such, this method bridges the statistical approaches taken by hypothesis testing techniques with the heuristic methods applied by system operators.

Besides the real-time data analyses, a database of component reliability can be utilized from which more accurate decisions can be made regarding equipment health. A list of some of the information that may be incorporated in the database is given in Table 2. The information employed for the second-stage system is derived from the general database, which also includes a tree-structured database as depicted in Figure 7. The tree-structured database is a linked list that facilitates the dynamic creation of system-specific rules from general relationships. It provides a physical description of the infrastructure of interest and knowledge of its instrumentation network, including sensor types, locations, manufacturers, model numbers, and nearby sensors. The tree-structured database is constructed to ensure that the fuzzy system is portable between diverse power plants and adaptable when either the sensory network or the infrastructure is modified. To reduce the possibility of false alarms due to common-cause instrument anomalies, the tree 


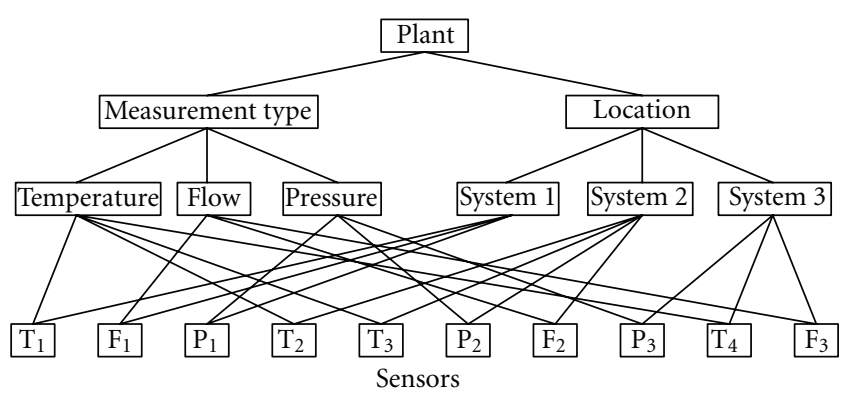

FIgure 7: The tree-structured database containing a physical description of the power plant and its sensory network.

structure may be used to search for the occurrence of similar abnormalities.

Another issue is whether the plant is operating at steadystate conditions or whether it is undergoing a transient, for instance, during startup or load changes. Some signal processing techniques are better suited to static conditions whereas other methods may be adept at dynamic conditions. Practically speaking, the data from base load nuclear power plants are generally easier to analyze than those from peaking units.

\section{Instrument Calibration Monitoring}

This section presents a more detailed example of the application of fuzzy logic to health monitoring of process instrumentation. Here we detail the use of fuzzy logic for a specific application, in particular by showing representative rules, fuzzy membership function, and actual results from the application of the FLDM to data from an operating nuclear power plant.

4.1. Calibration Reduction. In any process, the system conditions must be measured in order to achieve the desired operating configuration. Part of the effort in improving process monitoring, control, safety, and maintenance using automated computer systems must be dedicated to insuring the accuracy and reliability of the indicated process conditions as obtained from the instrumentation system [40]. Diagnostic functions and condition monitoring must be performed based on validated process signals. Sensor validation (or instrument fault detection) is a determination whether a process indicator is providing a reliable reading. The incentives for performing sensor validation lie in both concerns for safety and the economic returns possible. Properly validated signals increase plant availability and the reliability of operator actions.

Various approaches have been developed to perform signal validation. Many methods originated from the aerospace and nuclear industries. Most techniques employ a two-stage process: (1) generation of residuals and (2) decision making based upon hard thresholds. The residual signals (nominally near zero) deviate from zero in characteristic ways when certain faults occur. The residual generation methodologies include the following.
(1) Parity space (see review by Patton and Chen [41]),

(2) Dedicated observers $[42,43]$, and

(3) Kalman filters [44, 45].

The decision making is based upon various tests, including the generalized likelihood ratio (GLR) [46-48], sequential probability ratio test (SPRT), and innovation properties (tests for whiteness, mean, covariance, chi-square, etc.), applied to the residuals.

Two primary types of failure are addressed by sensor validation: (1) incipient (catastrophic) failure detection and (2) detection of instrument calibration drift. For instance, if instrument calibration is drifting over time, then preventive maintenance on the instrument channel may be performed before complete failure. This predictive maintenance aspect has the benefit of a maintenance scheduling aid, including the ability to preorder components, as well as the possibility in the severest case of preventing a nuclear plant trip, which depending on the nature could require the filing of a Licensee Event Report (LER) with the USA Nuclear Regulatory Commission-a situation to be avoided.

Calibration reduction is a particular application of sensor validation. Calibration reduction does not eliminate the need to perform instrument calibration, but lessens the effort involved. An obvious benefit of calibration monitoring is reduced manpower requirements due to the lessened workload. Since some sensors are normally inaccessible, this recalibration effort may come during an already busy plant outage-when labor is already at a premium. In a nuclear power plant, calibration reduction can also lower the amount of time spent in radiation areas, thereby reducing personnel exposure.

4.2. Fuzzy Logic Diagnostic Approach. Consider that multiple readings of a given process variable are available directly with physically redundant sensors and/or indirectly via analytical or empirical relationships. To begin the analysis, an absolute deviation between all possible signal pairings is calculated according to

$$
\delta_{i j}=\left|x_{i}-x_{j}\right| \quad \text { for } i=1,2, \ldots, N ; \quad i \neq j,
$$

where $N$ is the number of redundant sensors and $x_{i}$ is the measurement from the $i$ th sensor at a given time instant. The range of possible values, that is, the universe of discourse, for $\delta$ varies from zero, when the signal pair is in complete agreement, to the instrument range, $R$, which is the maximum possible deviation. This universe of discourse for $\delta$ is quantized into several overlapping fuzzy-set values:

$$
\begin{array}{cc}
S(\text { small } \delta): & {[0,5 \sigma]} \\
M(\text { medium } \delta): & {[3 \sigma, 9 \sigma]} \\
L(\text { large } \delta): & {[7 \sigma, R]}
\end{array}
$$

In particular, this deviation is normalized based on the signal standard deviation, $\sigma$, using three regions: (1) small, (2) medium, and (3) large deviations, as shown in Figure 8.

In performing instrument calibration monitoring, two results are important: (1) an instantaneous indication of how 


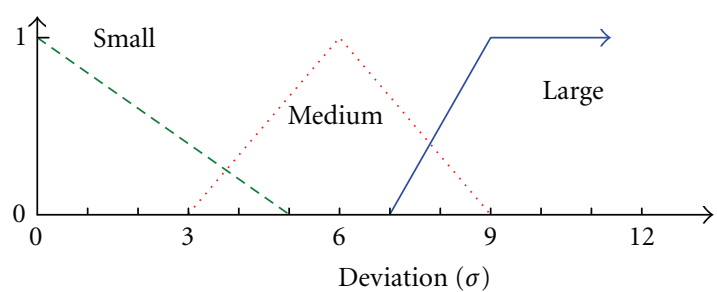

Figure 8: Universe of discourse for the deviation $(\delta)$ fuzzy input variable.

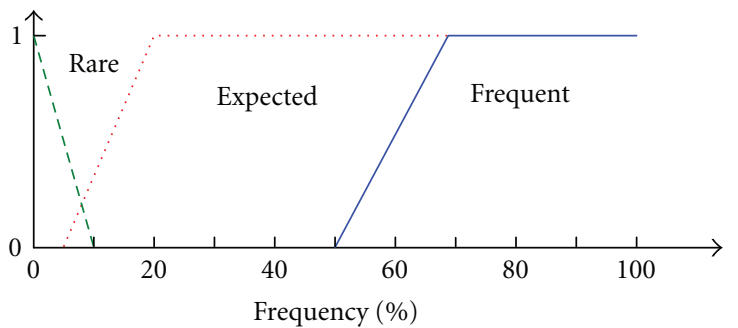

FIGURE 9: Universe of discourse for the frequency of occurrence $(\tau)$ fuzzy input variable.

a sensor is performing relative to its redundant units, and (2) a long-term determination of whether an instrument channel is functioning properly. The presence of random fluctuations requires a statistically significant number of data samples taken over a representative period to minimize the false alarm probability.

Therefore, not only must the sensor deviations be monitored, but also the frequency of the small, medium and large deviations. Hence, a second fuzzy input variable-the fre$q u e n c y, \tau^{k}$, at which the deviation type $k$ has been observedis introduced. In practice, the value of $\tau$ is obtained by maintaining a running average over a number of contiguous samples up to the current sample time, when the status decision is made. For $\tau$, the universe of discourse ranges from $0 \%$ to $100 \%$, and the fuzzy set values are (see Figure 9):

$$
\begin{array}{cc}
R(\text { rare }): & {[0 \%, 10 \%]} \\
E(\text { expected }): & {[5 \%, 100 \%]} \\
F(\text { frequent }): & {[50 \%, 100 \%]}
\end{array}
$$

Thus, there are three fuzzy frequency $(\tau)$ values for each deviation.

Rules are then implemented to determine whether the signals are consistent with respect to one another. The consistency checking algorithm may find several possible results:

(1) Signals Consistent: When all the signals are in agreement with one another, then either $(a)$ all the sensors are good, or $(b)$ all the instruments are out of calibration (e.g., due to signal drift).

(2) Signals Partially Consistent: If the majority of sensor readings are consistent with respect to one another, then the minority group of signals is suspected of having experienced a calibration shift. In fact, it

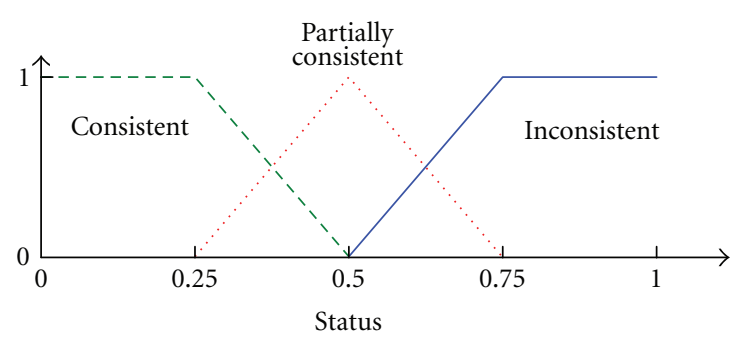

FIgure 10: Universe of discourse for the status $(s)$ fuzzy output variable.

is known that at least one sensor may need to be replaced or recalibrated, so maintenance may be planned accordingly.

(3) Signals Inconsistent: In this case, multiple sensors may require replacement or recalibration. It is in situations such as this (or in those instances where only one or two redundant signals exist) that an independent estimate of the process state is useful, that is, an analytical or empirical process model provides a virtual measurement with which to compare the recorded values.

The fuzzy validator therefore classifies a signal as being consistent, partially consistent, or inconsistent. The status fuzzy variable is the signal validity state, $s$, with fuzzy set values of

$$
\begin{array}{cc}
C(\text { consistent }): & {[0.0,0.5]} \\
P(\text { partiallyconsistent }): & {[0.25,0.75]} \\
I(\text { inconsistent }): & {[0.5,0.1]}
\end{array}
$$

which are graphed in Figure 10.

Rules associate the signal state with the deviation between the sensor readings, and the frequency at which those deviations have been observed. The rule set must be established such that a status result is issued for each signal. Furthermore, the rules are constructed to consider the effects that an inconsistent sensor has on the deviation and frequency of occurrence for a calibrated instrument. For example, in a signal pairing comparison between an accurate and an inaccurate reading, both sensors may initially be deemed inconsistent with respect to one another.

With a group of three or more sensors measuring the same state-variable, sensor fault isolation is possible. In the case of four redundant sensors, there are twelve state fuzzy variables and four fuzzy status variables. There are six deviations computed (e.g., $\delta_{12}$ and $\delta_{23}$ ), and eighteen frequency fuzzy variables (e.g., $\tau_{12}^{S}, \tau_{12}^{M}$ and $\tau_{12}^{L}$ ). The rules, which are shown in Table 3, are more extensive than those in the example of Section 3.2. The first rule in the table may be read as the set level implication.

If the deviation, $\delta_{12}$, is large and its frequency of occurrence, $\tau_{12}^{L}$, is not rare, then the sensor \#1 status, $s_{1}$, is inconsistent and the sensor \#2 status, $s_{2}$, is inconsistent.

Different weights are assigned for the rules. In particular, the first six rules use a weighting factor of 0.3 because of the adverse effect that a single faulty sensor can have on the consistency checking results for reliable instruments. Unit 
TABLE 3: Rules for the calibration monitoring of four sensors.

\begin{tabular}{|c|c|c|c|c|c|c|c|c|c|c|c|c|c|c|c|c|}
\hline$R$ & $\delta_{12}$ & $\tau_{12}$ & $\delta_{13}$ & $\tau_{13}$ & $\delta_{14}$ & $\tau_{14}$ & $\delta_{23}$ & $\tau_{23}$ & $\delta_{24}$ & $\tau_{24}$ & $\delta_{34}$ & $\tau_{34}$ & $s_{1}$ & $s_{2}$ & $s_{3}$ & $s_{4}$ \\
\hline 1 & $L$ & $\bar{R}$ & & & & & & & & & & & $I$ & $I$ & & \\
\hline 2 & & & $L$ & $\bar{R}$ & & & & & & & & & $I$ & & $I$ & \\
\hline 3 & & & & & $L$ & $\bar{R}$ & & & & & & & $I$ & & & $I$ \\
\hline 4 & & & & & & & $L$ & $\bar{R}$ & & & & & & $I$ & $I$ & \\
\hline 5 & & & & & & & & & $L$ & $\bar{R}$ & & & & $I$ & & $I$ \\
\hline 6 & & & & & & & & & & & $L$ & $\bar{R}$ & & & $I$ & $I$ \\
\hline 7 & $S$ & $F$ & & & & & & & & & & & $C$ & C & & \\
\hline 8 & & & $S$ & $F$ & & & & & & & & & $C$ & & $C$ & \\
\hline 9 & & & & & $S$ & $F$ & & & & & & & $C$ & & & $C$ \\
\hline 10 & & & & & & & $S$ & $F$ & & & & & & $C$ & C & \\
\hline 11 & & & & & & & & & $S$ & $F$ & & & & $C$ & & $C$ \\
\hline 12 & & & & & & & & & & & $S$ & $F$ & & & C & $C$ \\
\hline 13 & $M$ & $\bar{R}$ & & & & & & & & & & & $\bar{C}$ & $\bar{C}$ & & \\
\hline 14 & & & $M$ & $\bar{R}$ & & & & & & & & & $\bar{C}$ & & $\bar{C}$ & \\
\hline 15 & & & & & $M$ & $\bar{R}$ & & & & & & & $\bar{C}$ & & & $\bar{C}$ \\
\hline 16 & & & & & & & $M$ & $\bar{R}$ & & & & & & $\bar{C}$ & $\bar{C}$ & \\
\hline 17 & & & & & & & & & $M$ & $\bar{R}$ & & & & $\bar{C}$ & & $\bar{C}$ \\
\hline 18 & & & & & & & & & & & $M$ & $\bar{R}$ & & & $\bar{C}$ & $\bar{C}$ \\
\hline
\end{tabular}

weights are applied to Rules 7 through 12 since this situation of consistency has a high degree of confidence. The last six rules have a weight of 0.6 since their occurrence is to be expected given the statistical fluctuations present in a process and the variability of plant operating conditions.

The compactness of the rule base and the simplicity with which the deviations and frequency fuzzy variables are applied together with the rules allow signal processing in real time. The fuzzy logic approach is also capable of evaluating situations in which analytical models are employed. Deviations between analytical model estimates and hardware measurements can occur due to (1) a failure in the sensor measuring the state variable of interest or (2) an anomaly in an input signal sent to the analytical model.

The basis of the calibration reduction (but not total elimination) is the intercomparison of redundant process measurements. The process readings are monitored on a continuous basis - over weeks for instance. During this time period either the signals will agree or they will disagree. If (as one would hope), the redundant signals concur with one another, one of two conclusions can be surmised: (1) the signals are all accurate or (2) the signals have all failed by some common-cause mode such as calibration drift. The problem is then reduced to verification of the calibration of one of the redundant sensors (channels). Should the tested instrument prove to be within calibration specifications, it may be concluded that the remaining (untested) sensors are within calibration specifications and they do not require recalibration.

4.3. Calibration Reduction Example. The data shown in Figure 11 are pressurizer pressure measurements recorded at a commercial pressurized water reactor (PWR). Using the first 200 days worth of data, the deviations and the

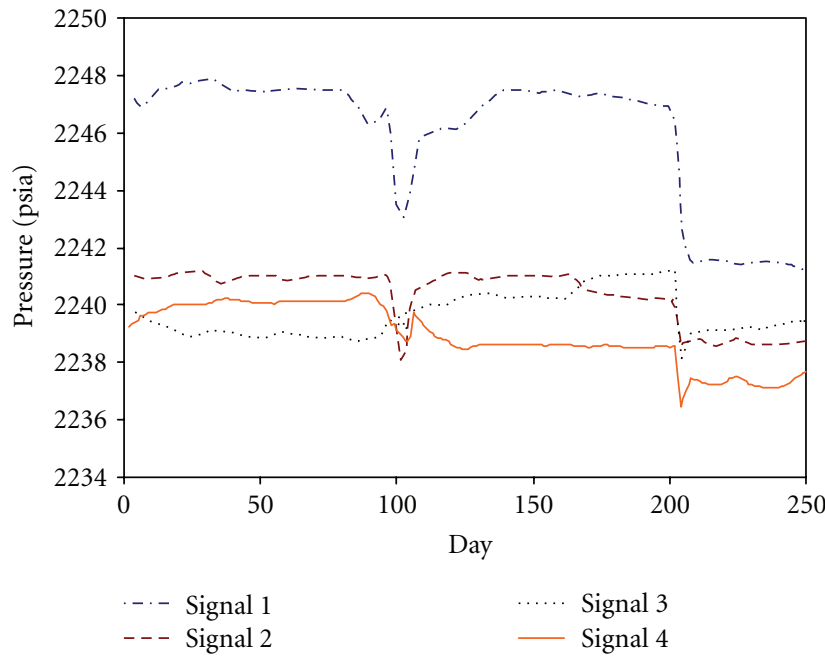

FIGURE 11: Pressurizer pressure measurements from four redundant sensors [49].

frequencies of occurrence for these pressure readings were first calculated and are given in Table 4. The fuzzified values are then evaluated using a Mamdani style fuzzy system, which employs minimum implication, summation aggregation, and centroid defuzzification. In the results for these pressurizer data, only six of the eighteen rules were activated. The fuzzy diagnostic monitor accurately identifies that Signal 1 is an out-of-calibration pressure transducer. Signal 2 is identified as consistent, and Signals 3 and 4 are deemed partially consistent. A close examination of Rules 1-3 and 10-12 reveals that this latter status is not entirely a consequence of the first signal's inconsistency, as Signals 2-4 are not tightly coupled as a group. The effect of the 
TABLE 4: Deviations and frequencies of occurrence computed for pressurizer pressure signals.

\begin{tabular}{|c|c|c|c|c|}
\hline \multirow{2}{*}{ Signal pair } & \multicolumn{2}{|c|}{ Before day 200} & \multicolumn{2}{|c|}{ After day 200} \\
\hline & Deviation $(\delta)$ & $\begin{array}{l}\text { Frequency of occurrence } \\
\qquad\left(\tau^{S}, \tau^{M}, \tau^{L}\right)\end{array}$ & Deviation $(\delta)$ & $\begin{array}{l}\text { Frequency of occurrence } \\
\qquad\left(\tau^{S}, \tau^{M}, \tau^{L}\right)\end{array}$ \\
\hline $1-2$ & 10.4 & $0 \%, 16 \%, 99 \%$ & 4.7 & $32 \%, 100 \%, 0 \%$ \\
\hline $1-3$ & 12.2 & $0 \%, 4.5 \%, 98 \%$ & 3.5 & $100 \%, 100 \%, 0 \%$ \\
\hline $1-4$ & 12.8 & $0 \%, 4 \%, 100 \%$ & 6.8 & $0 \%, 100 \%, 76 \%$ \\
\hline $2-3$ & 2.24 & $100 \%, 37 \%, 0 \%$ & 1.2 & $100 \%, 4 \%, 0 \%$ \\
\hline $2-4$ & 2.46 & $100 \%, 30 \%, 0 \%$ & 2.1 & $100 \%, 6 \%, 0 \%$ \\
\hline $3-4$ & 2.36 & $100 \%, 19 \%, 0 \%$ & 3.3 & $100 \%, 100 \%, 0 \%$ \\
\hline
\end{tabular}

inconsistent sensor can be removed by reapplying the fuzzy system to only Signals $2-4$. Neither the deviations nor the frequencies of occurrence have to be recomputed and a smaller, three-sensor rule set is used.

At approximately day 200, the faulty sensor was recalibrated such that it (visually speaking when looking at the data of Figure 11) becomes more consistent with the other three transducers. The particular deviations and frequencies of occurrence for the last 50 days are also given in Table 4. The fuzzy analysis results conclude that Signals 2 and 3 are consistent, and that Signals 1 and 4 are partially inconsistent. These results can be visually verified by noting that Signals 1 and 4 are the outliers in the data of Figure 11.

\section{Summary}

Predictive maintenance can be achieved without disturbing the plant operation. Fault-tolerant signal processing based on fuzzy logic can help power plant operators meet the goal of effective, efficient, and safe operation of complex systems. Fuzzy logic-based condition monitoring is described for improving state-of-the-art monitoring and diagnostic capabilities for general industrial processes. Its feasibility is shown through the analysis of sensor data obtained from operating reactor systems. Such technology has multidisciplinary applications besides electric generating stations, including chemical processing plants, highways, and air/space craft. In the longer term, FLDM could be provided with some level on online learning which might be augmented by operator input $[50,51]$.

\section{References}

[1] F. R. Rubio, M. Berenguel, and E. F. Camacho, "Fuzzy logic control of a solar power plant," IEEE Transactions on Fuzzy Systems, vol. 3, no. 4, pp. 459-468, 1995.

[2] A. Flores, D. Sáez, J. Araya, M. Berenguel, and A. Cipriano, "Fuzzy predictive control of a solar power plant," IEEE Transactions on Fuzzy Systems, vol. 13, no. 1, pp. 58-68, 2005.

[3] C. M. Leuba, M. Abdalla, C. E. Ford, and L. Guimaraes, "Hybrid fuzzy-PI adaptive control for U-tube steam generators," Control, Theory and Advanced Technology, vol. 8, no. 3, pp. 567-575, 1992.

[4] N. Na, K. Kwon, C. Ham, and Z. Bien, "A study on water level control of PWR steam generator at low power and the selftuning of its fuzzy controller," Fuzzy Sets and Systems, vol. 74, no. 1, pp. 43-51, 1995.
[5] G. Y. Park and P. H. Seong, "Application of a fuzzy learning algorithm to nuclear steam generator level control," Annals of Nuclear Energy, vol. 22, no. 3-4, pp. 135-146, 1995.

[6] G. Wang and K. E. Holbert, "Hybrid classical-fuzzy controller for a power plant water supply system," in Proceedings of the 34th Annual North American Power Symposium, pp. 352-359, Tempe, Ariz, USA, October 2002.

[7] M. Holmes and A. Ray, "Fuzzy damage-mitigating control of a fossil power plant," IEEE Transactions on Control Systems Technology, vol. 9, no. 1, pp. 140-147, 2001.

[8] G. P. Sullivan, R. Pugh, A. P. Melendez et al., Operations \& Maintenance Best Practices, a Guide to Achieving Operational Efficiency, Release 3. 0, Pacific Northwest National Laboratory, U.S. Department of Energy, Federal Energy Management Program, 2010.

[9] S. S. Choi, K. S. Kang, H. G. Kim, and S. H. Chang, "Development of an on-line fuzzy expert system for integrated alarm processing in nuclear power plants," IEEE Transactions on Nuclear Science, vol. 42, no. 4, pp. 1406-1418, 1995.

[10] K. E. Holbert, A. Mishra, and L. Mill, "Intrusion detection through SCADA systems using fuzzy logic-based state estimation methods," International Journal of Critical Infrastructures, vol. 3, no. 1-2, pp. 58-87, 2007.

[11] U. Pareek and I. N. Kar, "Estimating compressor discharge pressure of gas turbine power plant using type-2 fuzzy logic systems," in Proceedings of the IEEE International Conference on Fuzzy Systems, pp. 649-654, Vancouver, Canada, July 2006.

[12] B. Köeppen-Seliger, N. Kiupel, H. S. Kellinghaus, and P. M. Frank, "Fault diagnosis concept for a high-pressure-preheater line," in Proceedings of the 34th IEEE Conference on Decision and Control, pp. 2383-2388, New Orleans, La, USA, December 1995.

[13] M. G. Na, Y. J. Lee, and I. J. Hwang, "A smart software sensor for feedwater flow measurement monitoring," IEEE Transactions on Nuclear Science, vol. 52, no. 6, pp. 3026-3034, 2005.

[14] W. Yang, K. Y. Lee, S. T. Junker, and H. Ghezel-Ayagh, "Fuzzy fault diagnosis and accommodation system for hybrid fuelcell/gas-turbine power plant," IEEE Transactions on Energy Conversion, vol. 25, no. 4, pp. 1187-1194, 2010.

[15] R. Berrios, F. Núñez, and A. Cipriano, "Fault tolerant measurement system based on Takagi-Sugeno fuzzy models for a gas turbine in a combined cycle power plant," Fuzzy Sets and Systems, vol. 174, no. 1, pp. 114-130, 2011.

[16] P. M. Frank and B. Köppen-Seliger, "Fuzzy logic and neural network applications to fault diagnosis," International Journal of Approximate Reasoning, vol. 16, no. 1, pp. 67-88, 1997.

[17] L. F. Mendonça, J. M. Sousa, and J. M. G. Sá da Costa, "Fuzzy model-based fault detection and isolation," in Proceedings of 
the IEEE Conference on Emerging Technologies and Factory Automation, vol. 2, pp. 768-774, Lisbon, Portugal, September 2003.

[18] C.-C. Yu and C. Lee, "Fault diagnosis based on qualitative/ quantitative process knowledge," AIChE Journal, vol. 37, no. 4, pp. 617-628, 1991.

[19] C. Aubrun, D. Sauter, H. Noura, and M. Robert, "Fault diagnosis and reconfiguration of systems using fuzzy logic: application to a thermal plant," International Journal of Systems Science, vol. 24, no. 10, pp. 1945-1954, 1993.

[20] S. Amin, C. Byington, and M. Watson, "Fuzzy inference and fusion for health state diagnosis of hydraulic pumps and motors," in Proceedings of the Annual Meeting of the North American Fuzzy Information Processing Society (NAFIPS '05), pp. 13-18, June 2005.

[21] P. Vaija, M. Järveläinen, and M. Dohnal, "Multilevel failure detection system," Computers in Industry, vol. 6, no. 4, pp. 253-263, 1985.

[22] C. K. Mechefske, "Objective machinery fault diagnosis using fuzzy logic," Mechanical Systems and Signal Processing, vol. 12, no. 6, pp. 855-862, 1998.

[23] Y. Uriu, A. Ninomiya, Y. Kanda et al., "Monitoring of superconducting magnet system using fuzzy theorem," IEEE Transactions on Applied Superconductivity, vol. 5, no. 2, pp. 469-472, 1995.

[24] A. S. Heger, N. K. Alang-Rashid, and K. E. Holbert, "Use of fuzzy logic in signal processing and validation," Transactions of the American Nuclear Society, vol. 68, pp. 135-136, 1993.

[25] K. E. Holbert, A. Sharif Heger, and N. K. Alang-Rashid, "Redundant sensor validation by using fuzzy logic," Nuclear Science and Engineering, vol. 118, no. 1, pp. 54-64, 1994.

[26] A. S. Heger, K. E. Holbert, and A. M. Ishaque, "Fuzzy associative memories for instrument fault detection," Annals of Nuclear Energy, vol. 23, no. 9, pp. 739-756, 1996.

[27] G. Mourot, S. Bousghiri, and F. Kratz, "Sensor fault detection using fuzzy logic and neural networks," in Proceedings of International Conference on Systems, Man and Cybernetics, pp. 369-374, Le Touquet, France, October 1993.

[28] D. Sauter, N. Mary, F. Sirou, and A. Thieltgen, "Fault diagnosis in systems using fuzzy logic," in Proceedings of the $3 \mathrm{rd}$ IEEE Conference on Control Applications, vol. 2, pp. 883-888, Glasgow, UK, August 1994.

[29] J. W. Hines, D. J. Wrest, and R. E. Uhrig, "Signal validation using an adaptive neural fuzzy inference system," Nuclear Technology, vol. 119, no. 2, pp. 181-193, 1997.

[30] K. Lin and K. E. Holbert, "Design of a hybrid fuzzy classifier system for power system sensor status evaluation," in Proceedings of the IEEE Power Engineering Society General Meeting, pp. 1351-1358, San Francisco, Calif, USA, June 2005.

[31] G. Betta and A. Pietrosanto, "Instrument fault detection and isolation: state of the art and new research trends," IEEE Transactions on Instrumentation and Measurement, vol. 49, no. 1, pp. 100-107, 2000.

[32] V. Venkatasubramanian, R. Rengaswamy, K. Yin, and S. N. Kavuri, "A review of process fault detection and diagnosis part I: quantitative model-based methods," Computers and Chemical Engineering, vol. 27, no. 3, pp. 293-311, 2003.

[33] J. W. Hines and R. Seibert, Technical Review of on-Line Monitoring Techniques for Performance Assessment, Volume 1: State-of-the-Art, NUREG/CR-6895, U.S. Nuclear Regulatory Commission, 2006.

[34] R. J. Patton, P. M. Frank, and R. N. Clark, Issues of Fault Diagnosis for Dynamic Systems, Springer, 2000.
[35] R. Isermann, Fault-Diagnosis: An Introduction from Fault Detection to Fault Tolerance, Springer, 2006.

[36] M. C. Carnera, "Selection of diagnostic techniques and instrumentation in a predictive maintenance program. A case study," Decision Support Systems, vol. 38, no. 4, pp. 539-555, 2005.

[37] K. Lin and K. E. Holbert, "Blockage diagnostics for nuclear power plant pressure transmitter sensing lines," Nuclear Engineering and Design, vol. 239, no. 2, pp. 365-372, 2009.

[38] K. Lin and K. E. Holbert, "Void diagnostics in liquid-filled pressure sensing lines," Progress in Nuclear Energy, vol. 52, no. 5, pp. 503-511, 2010.

[39] K. Lin and K. E. Holbert, "Pressure sensing line diagnostics in nuclear power plants," in Nuclear Power, P. V. Tsvetkov, Ed., chapter 7, pp. 97-122, Sciyo, Rijeka, Croatia, 2010.

[40] K. E. Holbert and B. R. Upadhyaya, "Integrated signal validation system for nuclear power plants," Nuclear Technology, vol. 92, no. 3, pp. 411-427, 1990.

[41] R. J. Patton and J. Chen, "Review of parity space approaches to fault diagnosis," in Proceedings of the IFAC Fault Detection, Supervision and Safety for Technical Processes, pp. 65-81, September 1991.

[42] D. G. Luenberger, "Introduction to observers," IEEE Transactions on Automatic Control, vol. AC-16, no. 6, pp. 596-602, 1971.

[43] R. N. Clark, "Dedicated observer approach to instrument failure detection," in Proceedings of the 18th IEEE Conference on Decision and Control including the Symposium on Adaptive Processes, pp. 237-241, December 1979.

[44] J. C. Deckert, M. N. Desai, J. J. Deyst, and A. S. Willsky, "F8 DFBW sensor failure identification using analytic redundancy," IEEE Transactions on Automatic Control, vol. AC-22, no. 5, pp. 795-803, 1977.

[45] R. N. Clark and B. Campbell, "Instrument fault detection in a pressurized water reactor pressurizer," Nuclear Technology, vol. 56, no. 1, pp. 23-32, 1982.

[46] A. S. Willsky and H. L. Jones, "A generalized likelihood ratio approach to state estimation in linear systems subject to abrupt changes," in Proceedings of the IEEE Conference on Decision and Control, pp. 846-853, 1974.

[47] J. L. Tylee, "On-line failure detection in nuclear power plant instrumentation," IEEE Transactions on Automatic Control, vol. AC-28, no. 3, pp. 406-415, 1983.

[48] S. Narasimhan and R. S. H. Mah, "Generalized likelihood ratios for gross error identification in dynamic processes," AIChE Journal, vol. 34, no. 8, pp. 1321-1331, 1988.

[49] K. E. Holbert and H. M. Hashemian, "Instrument calibration reduction using signal validation," Transactions of the American Nuclear Society, vol. 69, pp. 372-373, 1993.

[50] K. E. Holbert and X. Wang, "Power plant diagnostic information fusion using fuzzy logic," in Proceedings of the World Automation Congress Intelligent Automation and Soft Computing, vol. 1, pp. ISIAC044. 1-ISIAC044. 6, Anchorage, Alaska, USA, May 1998.

[51] X. Wang and K. E. Holbert, "A fuzzy logic enhanced fault diagnostic system," in Proceedings of the Maintenance and Reliability Conference (MARCON '97), vol. 2, pp. 92. 01-92. 15, Knoxville, Tenn, USA, May 1997. 

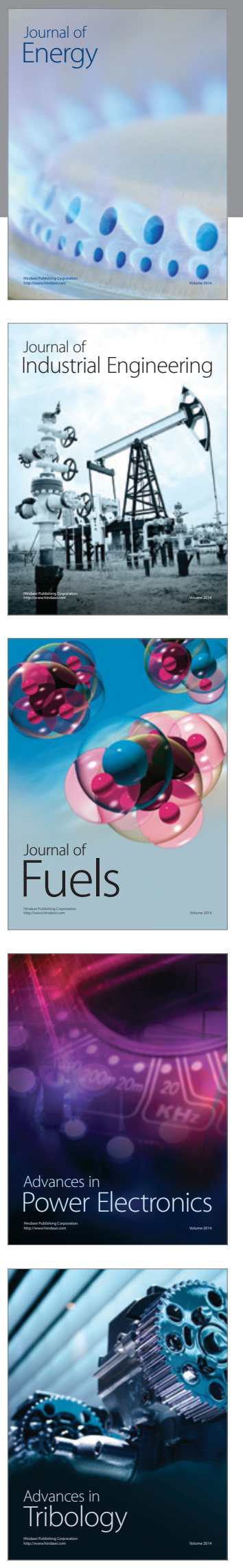
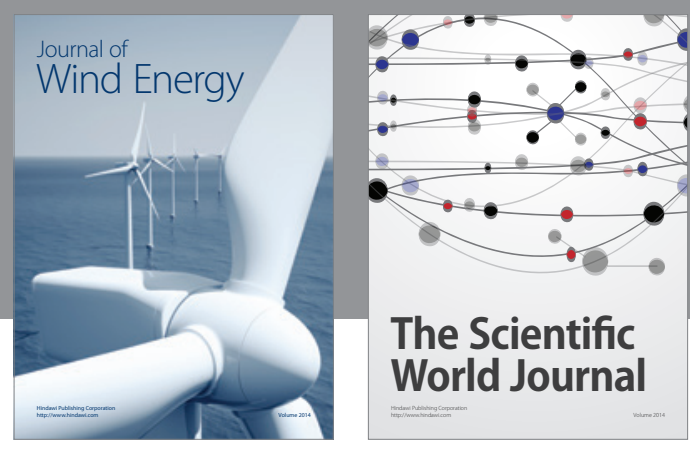

The Scientific World Journal

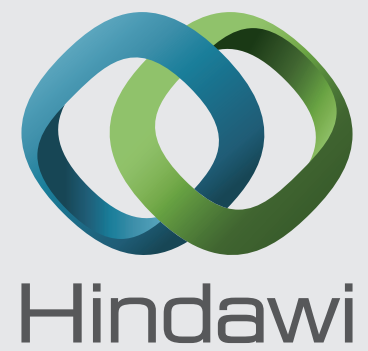

Submit your manuscripts at http://www.hindawi.com
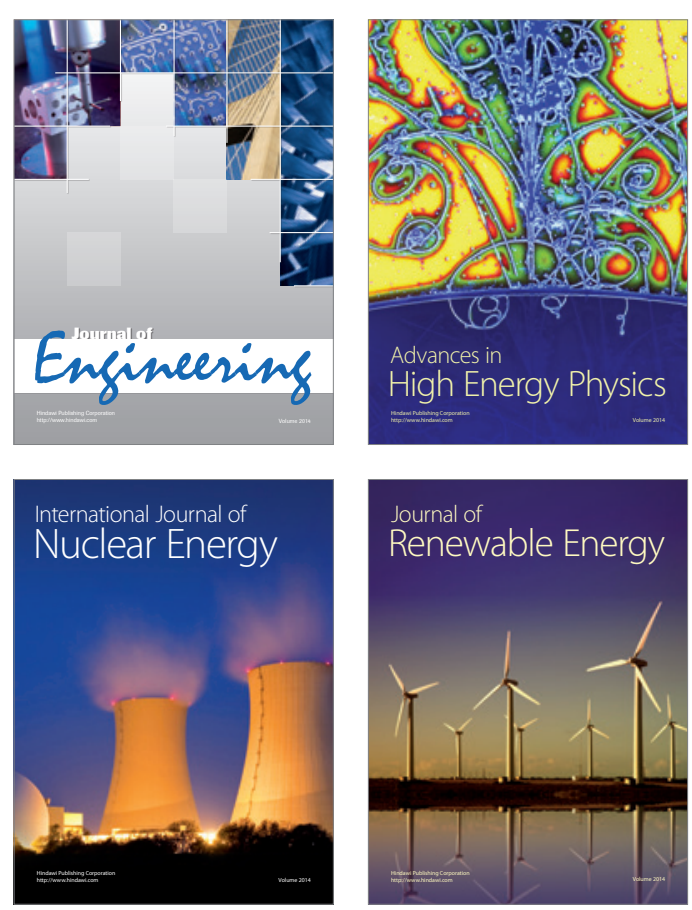

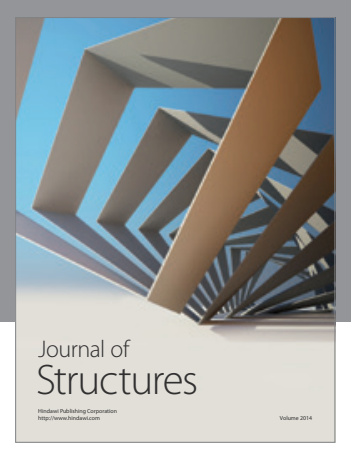

Rotating
Mechinery
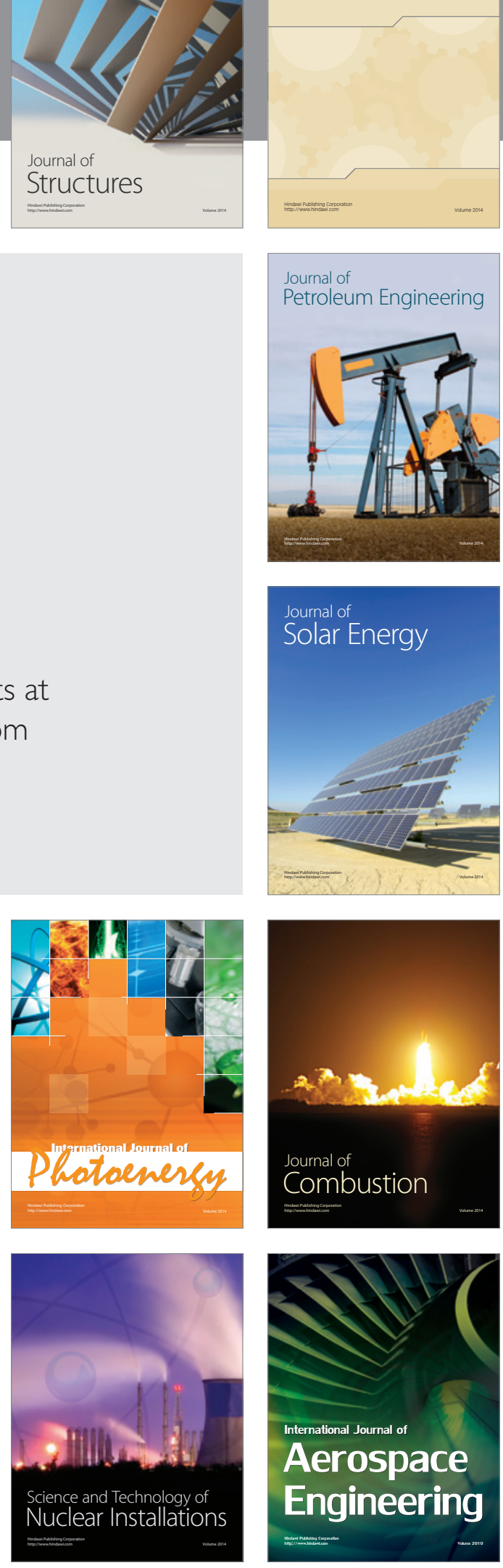